\title{
Dental student combines studies with experimental art
}

Fourth year Queen's University Belfast dental student Magdalena Wojcik has created a piece of artwork (pictured here) inspired by her career of choice.

Magdalena said: 'The inspiration initially came from the structure of crystal rocks and nature. However, during the process of creating it, I noticed the similarity of the different layers of rocks to the different layers in tooth structure. This was quite a funny discovery as I ended up creating a piece of art which looks exactly like what I look at every day: teeth.
'I love to experiment with a variety of media during my creative process. I started off by using solely acrylic paint, however now my paintings consist of broken glass, quartz crystals, acrylic paint and resin (art resin not composite). I love the $3 \mathrm{D}$ effect that this gives, and the shiny glass-like appearance.'

Magdalena regularly creates art and says that this contributed to her decision to choose dentistry as a career. 'I believe that dentistry is a form of art, in that we have to create a smile for the patient. It really takes an artist's eye to choose the correct shape, tooth length and colour to provide the best aesthetic outcome for the patient. On top of that for me, art is a form of release. It allows me to clear my head which I know a lot of people struggle with. That is why a hobby is really helpful in such an intense career.'

Magdalena, who is also the BDA student representative for her university, is currently leasing her artwork with some local business owners in Northern Ireland for display in their spaces.

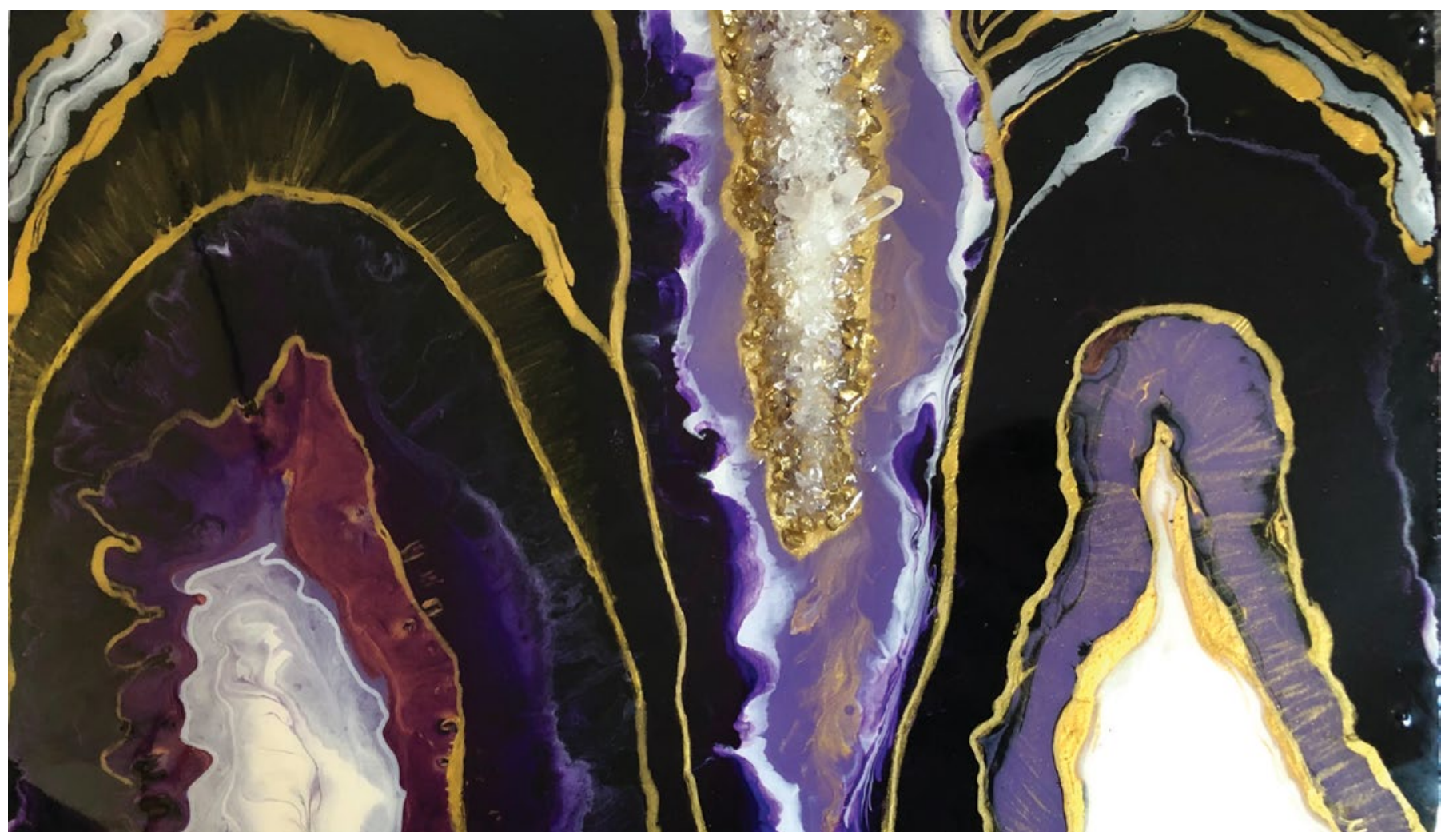

\section{Nationwide engagement on future dental services}

A widespread engagement exercise is getting underway to ensure the workforce delivering future dental services meets the needs and expectations of patients and the priorities set out in the NHS Long Term Plan.

The exercise builds on work already carried out by Health Education England (HEE) as part of the three-year Advancing Dental Care Review.

The review, led by HEE's seven dental deans, aims to identify the skills, training, workforce profile and funding models needed to meet future population demands.
It is due to make its recommendations in 2020/21 following a roll-out of new pilot training models.

Starting in October and continuing until December, engagement will take place with national and regional stakeholders ranging from public and professional bodies, employers and dental schools to local practices and commissioners.

The public's views will be gathered via local patient representatives and Healthwatch groups.

Professor Simon Gregory, HEE’s deputy medical director, primary and integrated care, said: 'As with most health services, the dental workforce needs to adapt to meet the changing needs of the twenty-first century. This England-wide review will help us create flexible training opportunities to enhance recruitment and retention within the NHS, develop new skill-mix models and address geographical and dental workforce shortages'.

Key issues already identified by the project include workforce optimisation and an unequal distribution of training places in England across the dental specialities. 